the risk was greatest in the first year. Factors related by multivariate analysis to relapse were neurologic abnormalities and organic etiology, mental retardation, seizure type (infantile spasms, absence seizures), and appearance or persistence of EEG abnormalities during the course of the illness and before discontinuation of the drugs. (Matricardi M et al. Outcome after discontinuation of antiepileptic drug therapy in children with epilepsy. Epilepsia October 1989; 30:582-589).

COMMENT. The authors believe that drug withdrawal can be at empted in patients with well controlled idiopathic epilepsy, without signs of brain damage and without persistent EEG abnormalities. They stress that predictive factors must be considered to individualize the risk of relapse for each patient.

\title{
METHYSERGIDE AND INFANTILE SPASMS
}

A trial of antiadrenergic and antiseretonergic drugs in the treatment of 24 newly diagnosed and previously untreated infantile spasm patients is reported from the Epilepsy Research Center, Section of Neurophysiology, Department of Neurology, Baylor College of Medicine; the Methodist Hospital; and Texas Children's Hospital; Houston, TX. Response to therapy was determined with 24 hour polygraphic/video monitoring techniques and was defined as complete control of spasms and disappearance of the hypsarrhythmic EEG pattern. Two of 12 patients treated with alpha-methylparatyrasene and one of 12 treated with methysergide showed a response. (Hrachovy RA et al. Treatment of infantile spasms with methysergide and alpha-methylparatyrasene. Epilepsia October 1989; 30:607-610).

COMMNT. The hypothesis that infantile spasms may result fram a dysfunction of monoaminonergic neurotransmitter systems is not exactly confirmed by the results of this study. However, the authors were impressed that the patients responding to treatment were not in spontaneous remission. Methysergide cannot displace $\mathrm{ACIH}$ as the treatment of choice in infantile spasms.

\section{NUIRI'TIONAL DEFICIENCIES AND SEIZURES}

\section{BIOTIN RESPONSIVE ENCEPHAIOPATHY}

A case of biotin responsive infantile encephalopathy is reported from the Department of Pediatrics and Child Neuropsychiatry, University of Verona, Italy; and Hcpital des Enfants Malades, Paris, France. At one month of age the infant developed dermatitis of the ears. At two months she began to have tonic clonic seizures occurring several times a day and refractory to treatment with carbamazepine, phenobarbital, phenytoin, clonazepam, nitrazepam, ACTH, and hydrocortisone. Seizure frequency increased up to ten per day. At three months, she became hypotonic and a CT scan showed enlargement of cortical sulci and lateral ventricles. At four months she was very lethargic and floppy, reflexes were hyperactive, and plantar responses were extensor. Her behavior was autistic-like and her scalp hair was sparse. The urine examination 